\title{
Implications of Inconsistencies between fMRI and dMRI on Multimodal Connectivity Estimation
}

\author{
Bernard $\mathrm{Ng}^{1,2}$, Gael Varoquaux ${ }^{1}$, Jean Baptiste Poline ${ }^{1}$, and Bertrand Thirion ${ }^{1}$ \\ ${ }^{1}$ Parietal team, Neurospin, INRIA Saclay, France \\ ${ }^{2}$ FIND Lab, Stanford University, United States \\ bernardyng@gmail.com
}

\begin{abstract}
There is a recent trend towards integrating resting state functional magnetic resonance imaging (RS-fMRI) and diffusion MRI (dMRI) for brain connectivity estimation, as motivated by how estimates from these modalities are presumably two views reflecting the same underlying brain circuitry. In this paper, we show on a cohort of 60 subjects that conventional functional connectivity (FC) estimates based on Pearson's correlation and anatomical connectivity (AC) estimates based on fiber counts are actually not that highly correlated for typical RS-fMRI ( $\sim 7 \mathrm{~min})$ and dMRI ( $\sim 32$ gradient directions) data. The FC-AC correlation can be significantly increased by considering sparse partial correlation and modeling fiber endpoint uncertainty, but the resulting FC-AC correlation is still rather low in absolute terms. We further exemplify the inconsistencies between $\mathrm{FC}$ and $\mathrm{AC}$ estimates by integrating them as priors into activation detection and demonstrating significant differences in their detection sensitivity. Importantly, we illustrate that these inconsistencies can be useful in fMRI-dMRI integration for improving brain connectivity estimation.
\end{abstract}

Keywords: brain activation, connectivity, dMRI, fMRI, multimodal integration.

\section{Introduction}

Recently, there is a growing interest in integrating resting state functional magnetic resonance imaging (RS-fMRI) and diffusion MRI (dMRI) for brain connectivity estimation $[1,2]$. This is motivated by how connectivity inferred from these modalities are presumably two views resembling the same underlying wiring structure of the brain. To enable meaningful integration, it is important to understand the relationships between brain function and structure and the degree to which these relationships are reflected by the connectivity estimates derived from RS-fMRI and dMRI data. A number of studies suggest a strong positive correlation between RS-fMRI and dMRI connectivity estimates [3-5], but it is unclear how much this correlation depends on the data acquisition and analysis methods.

Pearson's correlation is, by far, the most widely-used estimate of functional connectivity (FC) for RS-fMRI studies. Due to confounds, such as head motions, scanner noise, and physiological artefacts, as well as the typically small sample size as limited by data acquisition rate of standard pulse sequences, Pearson's correlation is subject 
to a high degree of estimation error [6]. Also, Pearson's correlation cannot distinguish direct from indirect connections, which complicates connection structure identification [6]. As for inferring anatomical connectivity (AC) from dMRI data, fiber count and the average fractional anisotropy (FA) along fiber tracts between pairs of brain areas are typically used as estimates [7]. Approaches for estimating the probability of diffusion between brain area pairs have been put forth [7], but do not scale well computationally to whole-brain analysis. In general, the fundamental limitations of fiber tractography pose great challenges to accurate AC estimation [7], especially when applied to typical dMRI data with limited number of gradient directions due to acquisition time restrictions. Besides the problem of crossing fibers, which harms the accuracy of the fiber tracts' trajectories, the location of the fiber endpoints holds high uncertainty, since the diffusion orientation is ambiguous near white-gray matter interface and tractography often terminates before reaching gray matter tissues [7].

In this paper, we assess the impact of the aforementioned limitations in data acquisition and analysis techniques on the consistency of FC and AC estimates. On typical RS-fMRI ( 7 min) and dMRI (32 gradient directions) data collected from a cohort of 60 subjects, we take the correlation between conventional FC and AC estimates based on Pearson's correlation and fiber count as baseline, and examine the effects of using sparse partial correlation [8] as the FC estimate, which controls for estimation errors and reduces indirect influences. We also propose a simple post-processing scheme for modeling fiber endpoint uncertainty and examine its influence on the FC-AC correlation. These comparisons are performed to determine whether and to what extent methodological improvements can compensate for limitations in data acquisition. In addition, we describe how these FC and AC estimates can be meaningfully incorporated as priors for task activation detection to further evaluate their consistency. The implications of combining the FC and AC estimates are also explored.

\section{Methods}

In this work, we are interested in contrasting Pearson's correlation against sparse partial correlation as FC estimates (Section 2.1) and examining the effects of modeling the uncertainty in fiber endpoint location when estimating $\mathrm{AC}$ (Section 2.2). Consistency between various FC and AC estimates is evaluated based on their correlation and their impact as priors on task activation detection (Section 2.3).

\subsection{Functional Connectivity Estimation}

Pearson's Correlation. Let $\mathbf{Z}$ be a $t \times d$ matrix with normalized RS-fMRI time courses (i.e. demeaned with unit standard deviation) of $d$ brain areas along the columns. The Pearson's correlations between all brain area pairs are given by: $\mathbf{C}=\mathbf{Z}^{\mathrm{T}} \mathbf{Z} /(t-1)$. For typical RS-fMRI data, $t<d$, thus $\mathbf{C}$ will contain high estimation errors [6]. Also, elements in $\mathbf{C}$ are assumed independent from each other, which renders separation of direct connections from indirect connections impossible [6]. These limitations can be alleviated by adopting sparse partial correlation as an FC estimate, as discussed next. 
Sparse Partial Correlation. Given a $d \times d$ empirical covariance matrix, $\mathbf{S}$, computed from samples drawn from a centered multivariate Gaussian distribution, we can estimate a well-conditioned sparse invariance covariance matrix, $\hat{\boldsymbol{\Lambda}}$, by minimizing the negative log-likelihood over the space of positive definite matrices, $\boldsymbol{\Lambda}>0$, and imposing an $l_{1}$ penalty on the off diagonal elements of $\boldsymbol{\Lambda}[8]$ :

$$
\min _{\mathbf{\Lambda}>0} \operatorname{tr}(\mathbf{S} \boldsymbol{\Lambda})-\log \operatorname{det}(\mathbf{\Lambda})+\lambda\|\mathbf{\Lambda}\|_{1}
$$

Sparse partial correlation, $\hat{\boldsymbol{\Gamma}}$, can then be computed by applying a simple normalization on $\hat{\boldsymbol{\Lambda}}: \hat{\boldsymbol{\Gamma}}_{i j}=-\hat{\boldsymbol{\Lambda}}_{i j} / \sqrt{\hat{\boldsymbol{\Lambda}}_{i i} \hat{\boldsymbol{\Lambda}}_{j j}}$ for $i \neq j$ and $\hat{\boldsymbol{\Gamma}}_{i j}=1$ for $i=j$. The level of sparsity on $\hat{\Lambda}$ is governed by $\lambda$, which we select using a refined grid search strategy combined with cross-validation as in [1]. (1) can be efficiently solved using e.g. the QUadratic Inverse Covariance (QUIC) algorithm [8]. In the context of FC estimation, $\mathbf{S}$ corresponds to $\mathbf{C}$ of a given subject. We highlight that enforcing sparsity serves the dual purpose of controlling for estimation errors and imposing our prior knowledge that the connection structure of the brain is sparse [1]. Also, partial correlation provides a measure of connectivity between two brain areas with the effects from all other brain areas partialled out, thus discriminates direct connections from indirect connections.

\subsection{Anatomical Connectivity Estimation}

The fiber count between a pair of brain areas is widely-used as an estimate of AC, since this measure presumably reflects the anatomical capacity for functional interactions. This $\mathrm{AC}$ estimate requires the fiber tracts to terminate in gray matter brain areas. For terminating a fiber tract, low FA (e.g. 0.15) and large curvature (e.g. $45^{\circ}$ ) are typically used as the criteria [7]. Although gray matter tissues have relatively lower FA than white matter tissues, voxels containing crossing fibers or near white-gray matter boundaries also have low FA. Thus, fiber tracts might not necessarily terminate in gray matter voxels. One simple way to deal with this limitation is to extrapolate along the tangent direction of the fiber endpoints (e.g. $<10 \mathrm{~mm}$ from the endpoints) until the fiber tracts reach a gray matter voxel (Fig. 1(a), option 2). However, the location of the resulting endpoints could very well be incorrect, especially if the original fiber endpoints are near gyri (Fig. 1(a)) [7]. In fact, the restriction on curvature during tractography would bias fiber tracts towards option 2 in Fig. 1(a) if tracking is not prematurely terminated by low FA [7].

To model the uncertainty in fiber endpoint location, we propose the following schem: Let $\boldsymbol{\omega}^{p}$ be a $27 \times 1$ weight vector associated with an endpoint $p$ of a fiber tract. We assign a weight of 1 to the endpoint and a weight of $\exp \left(-\Delta_{p s}\right)$ to its 26-connected neighbors but only if the neighbors are gray matter voxels. If no neighboring gray matter voxels are present, the given fiber is discarded. $\Delta_{p s}$ is the distance between the endpoint $p$ and the neighbor $s$ in voxel units. We then normalize $\boldsymbol{\omega}^{p}$ such that its 
elements sum to 1 . The same procedure is performed for the other endpoint $q$ of the given tract in generating $\boldsymbol{\omega}_{q}$. For each pair of elements $\boldsymbol{\omega}^{p}(k)$ and $\boldsymbol{\omega}^{q}(l)$ in which the two corresponding voxels lie in brain area $i$ and $j$, respectively, we add $\boldsymbol{\omega}^{p}(k) \cdot \boldsymbol{\omega}^{q}(l)$ to $\mathbf{A}_{i j}$, where $\mathbf{A}_{i j}$ is the $(i, j)^{\text {th }}$ element of the fiber count matrix, $\mathbf{A}$. In effect, we are placing a Gaussian kernel at each endpoint and partitioning a tract across spatially proximal brain areas to model endpoint uncertainty. If all neighbors of endpoint $p$ lie within the same brain area $i$ and all neighbors of endpoint $q$ lie within the same brain area $j$, the scheme above reduces to simple fiber count. Note that the resulting AC estimates would depend on the choice of neighborhood size and the drop off rate of the Gaussian kernel. We defer sensitivity analysis on these parameters to future work.

\subsection{Connectivity Consistency Assessment}

We evaluate the consistency between the various FC and AC estimates described in Section 2.1 and 2.2 using two criteria. The first is simply the correlation between the FC and AC estimates averaged over subjects. The second is based on the incorporation of the FC and AC estimates as priors for task activation detection using the model that we proposed in [9]. The assumption is that if the FC and AC estimates are consistent, then using them as priors should result in the same brain areas detected as activated. To safely base our assessment on activation detection, we employed the maximum-t permutation test [10] to enforce strict control on false positive rate. Under the model in [9], the maximum a posteriori mean of the activation effects is given by:

$$
\mathbf{M}=\left(\mathbf{V}_{1}^{-1}+\alpha \mathbf{V}_{2}^{-1}\right)^{-1} \mathbf{V}_{1}^{-1} \mathbf{Y} \mathbf{X}^{T}\left(\mathbf{X} \mathbf{X}^{T}\right)^{-1}
$$

where $\mathbf{Y}$ is a $d \times n$ matrix containing task fMRI time courses of $d$ brain areas of a given subject. $\mathbf{X}$ is a $m \times n$ regressor matrix, where $m$ is the number of experimental conditions. $\mathbf{V}_{1}$ and $\mathbf{V}_{2}$ are $d \times d$ covariance matrices of $\mathbf{Y}$ and the activation effects, respectively. We assume $\mathbf{V}_{1}=I_{d \times d}$ as conventionally done and $\mathbf{V}_{2}$ is where we inject different connectivity priors. The influence of the prior on $\mathbf{M}$ is controlled by $\alpha$, which we optimize based on model evidence [9]. We note that the model in [9] assumes $\mathbf{V}_{2}^{-1}$ is positive definite, hence theoretically, only FC estimates, $\hat{\boldsymbol{\Lambda}}$, generated by (1) can be directly employed for this model. In practice, even if $\mathbf{V}_{2}^{-1}$ is only positive semi-definite, with $\mathbf{V}_{1}=I_{d \times d}$, the optimal $\alpha$ derived based on model evidence would ensure $V_{1}^{-1}+\alpha V_{2}^{-1}$ is invertible. This observation is particularly important, since as we will now show, it enables fiber count, ill-conditioned Pearson's correlation matrix, and combinations of these connectivity estimates to be integrated into activation effect estimation. Specifically, let $\mathbf{W}$ be a $d \times d$ matrix with non-negative elements, $\mathbf{W}_{i j}$, reflecting the connectivity between brain areas $i$ and $j$, and $\mathbf{L}=\mathbf{D}$ $\mathbf{W}$ be the corresponding weighted graph Laplacian, which is always positive semidefinite. $\mathbf{D}$ is a $d \times d$ diagonal matrix with $\mathbf{D}_{i i}=\Sigma_{j} \mathbf{W}_{i j}$. We claim here that $\mathbf{L}$ is a useful prior that permits connectivity estimates to be meaningfully integrated into activation effect estimation when substituted into $\mathbf{V}_{2}^{-1}$. For intuition, consider the simplified scenario in which $m=1$ and the columns of $\mathbf{X}$ are orthonormal. One can easily show 
that the negative log posterior distribution of the activation effects comprises a term $\mathbf{a}^{\mathrm{T}} \mathbf{L a}$, where a corresponds to activation effects. Since $\mathbf{a}^{\mathrm{T}} \mathbf{L a}=\left(\Sigma_{i j}\left(\mathbf{a}_{i}-\mathbf{a}_{j}\right)^{2} \mathbf{W}_{i j}\right) / 2$, minimizing the negative log posterior probability penalizes discrepancies between the activation effect estimates of connected brain areas. Given this setup, AC estimates can be easily integrated into activation effect estimation by setting $\mathbf{W}$ to fiber count. For Pearson's correlation, $\mathbf{C}$, one would normally take the matrix inverse and directly assign it to $\mathbf{V}_{2}^{-1}$. However, since $t<d$ for typical RS-fMRI data, matrix inversion would be unstable. We thus propose zeroing the negative elements of $\mathbf{C}$ and using the resulting matrix as $\mathbf{W}$. The rationale is that negative FC estimates are usually associated with less anatomical support, i.e. lower fiber count, hence we hypothesize that they are more likely to be false correlations [5]. Building on this intuition, further zeroing elements of $\mathbf{C}$ with no anatomical support might help remove some of the noise-induced correlations. Conversely, spurious fiber tracts induced by tractography errors have no functional relevance. Thus, retaining only elements of the fiber count matrix with functional support based on the sparsity pattern of $\hat{\boldsymbol{\Lambda}}$ might improve activation detection. We assess the effectiveness of these simple FC-AC integration schemes by setting $\mathbf{W}$ to the corresponding connectivity estimates.

\section{$3 \quad$ Materials}

60 healthy subjects were recruited and scanned at multiple centers. Each subject performed 10 experimental tasks similar to those in [11], as fMRI data were acquired over a duration of $\sim 5$ min. $\sim 7$ min of RS-fMRI data were also collected. Scanning was performed using $3 \mathrm{~T}$ scanners from multiple manufacturers with $\mathrm{TR}=2200 \mathrm{~ms}$, TE $=$ $30 \mathrm{~ms}$, and flip angle $=75^{\circ}$. The task fMRI data were corrected for slice timing and head motions, temporally detrended, and spatially normalized using the SPM8 software. The RS-fMRI data were similarly preprocessed except a band-pass filter with cutoff frequencies at 0.01 to $0.1 \mathrm{~Hz}$ was applied. White matter and cerebrospinal fluid confounds were regressed out from the gray matter voxel time courses.

We divided the brain into $N=500$ parcels by concatenating RS-fMRI time courses across subjects and applying hierarchical Ward clustering. The gray matter voxel time courses within each parcel were averaged to generate parcel time courses. The time courses were then normalized by subtracting the mean and dividing by the standard deviation to account for scanner variability across imaging centers. The choice on $N$ was based on a recent brain surface analysis and macaque monkey studies [12]. From the perspective of stable FC estimation with limited time samples, setting $N$ to a lower value would be more suitable. We defer investigation on how to draw a balance between functional localization and stable FC estimation for future work.

dMRI data were collected from the same 60 subjects with $\mathrm{TR}=15000 \mathrm{~ms}$, TE $=$ $104 \mathrm{~ms}$, flip angle $=90^{\circ}, 32$ gradient directions, and b-value $=1300 \mathrm{~s} / \mathrm{mm}^{2}$. After correcting for eddy currents and head motions using FSL, tensor estimation and fiber tractography based on a single-tensor model were performed using MedINRIA. To better deal with crossing fibers, we also employed the unscented Kalman filter (UKF) tractography algorithm with a two-tensor model [13] for comparison. We warped our parcel template onto each subject's $\mathrm{B}_{0}$ volume for fiber count computation. 
(a)

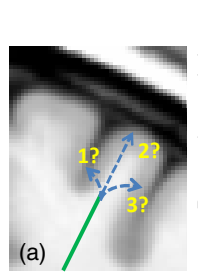

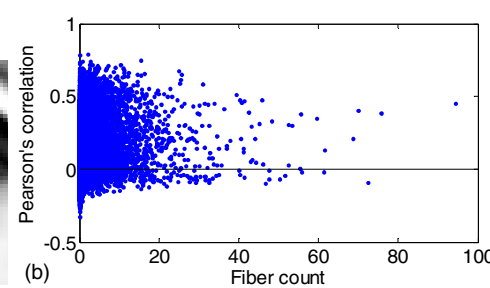

Table 1. Correlation between FC and AC estimates

\begin{tabular}{|l|l|c|c|}
\hline \multicolumn{2}{|l|}{$\begin{array}{l}\text { Endpoint } \\
\text { Processing }\end{array}$} & $\begin{array}{c}\text { Pearson's } \\
\text { correlation }\end{array}$ & $\begin{array}{c}\text { Sparse partial } \\
\text { correlation }\end{array}$ \\
\hline (c) & $\begin{array}{l}\text { Extrapolate } \\
\text { along tangent }\end{array}$ & 0.1223 & 0.1347 \\
\hline & $\begin{array}{l}\text { Gaussian } \\
\text { kernel }\end{array}$ & 0.1455 & 0.1538 \\
\hline & $\begin{array}{l}\text { Extrapolate } \\
\text { along tangent }\end{array}$ & 0.0530 & 0.0591 \\
\hline $\begin{array}{l}\text { Gaussian } \\
\text { kernel }\end{array}$ & 0.0731 & 0.0779 \\
\hline
\end{tabular}

Fig. 1. Connectivity estimate comparisons. (a) Fiber endpoint near gyri has high uncertainty. (b) Pearson's correlation vs. fiber count. (c) Correlation between FC and AC estimates.

\section{Results and Discussion}

The correlation between various FC and AC estimates are summarized in Fig. 1(c). Fiber count based on tracts generated by MedINRIA with endpoint extrapolation is taken as the baseline AC estimate. Conventional Pearson's correlation and fiber count are not very consistent (Fig. 1(b)) with an average correlation of only 0.1223 across subjects, which is much lower than that reported in [4]. In fact, for the same estimated AC, the estimated FC vary substantially. This finding suggests a high variability in the degree of functional interactions for the same anatomical capacity, but could also be attributed to limitations of the conventional connectivity estimates and data acquisition. Using sparse partial correlation as an FC estimate to control for estimation errors and indirect effects significantly increased the FC-AC correlation based on a Wilcoxon signed rank test ( $\mathrm{p}$-value $<0.01$ ). Modeling fiber endpoint uncertainty further increased the FC-AC correlation ( $\mathrm{p}$-value $<0.01$ ), but the overall correlation is still low compared to [4], in which RS-fMRI data of 35 min and dMRI data with 128 gradient directions were acquired from 5 subjects. Our results thus indicate that methodological improvements might not be adequate to compensate for the fundamental limitations in data acquisition. Further examining this point, we employed UKF tractography, which exploits tracking history to improve fiber estimation [13], but the resulting FC-AC correlation was lower than MedINRIA. We suspect that the number of gradient directions in our data was insufficient to benefit from UKF tractography.

FC-AC consistency results with activation detection as the assessment criterion are shown in Fig. 2. We used maximum-t permutation [10] to strictly control false positive rate, so that more detections would imply higher sensitivity. The average percentage of parcels detected over 10 experimental conditions and 21 contrasts between these conditions using ordinary least square (OLS) is taken as the baseline. Integrating FC estimated using Pearson's correlation significantly increased sensitivity (Fig. 2(a)) based on a permutation test described in [1]. Minor improvement over Pearson's correlation was observed at lower p-value thresholds by incorporating AC priors with tracts extracted using UKF tractography. A greater increase in sensitivity was found using AC priors with tracts generated by MedINRIA, which was slightly improved by modeling endpoint uncertainty. Overall, highest sensitivity was achieved with sparse inverse covariance as an FC prior. These differences in detection sensitivity again 

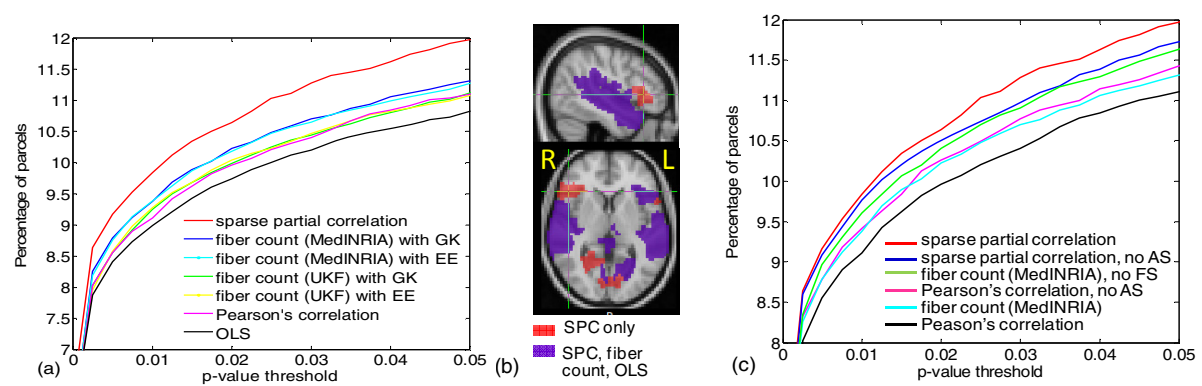

Fig. 2. Activation detection results. (a) $\%$ of parcels found activated vs. p-values with FC and AC estimates separately incorporated as priors. GK $=$ Gaussian kernel, EE $=$ endpoint extrapolation. (b) Activation map for an auditory task, p-value $<0.01$. SPC $=$ sparse partial correlation. (c) FC and AC estimates combined. AS = anatomical support, FS = functional support.

indicate that the FC and AC estimates are not very consistent. In fact, using AC priors generally detected less distant bilateral activation (Fig. 2(b)), which is likely due to difficulties in tracking fibers across the two hemispheres [7]. Removing Pearson's correlation with no anatomical support significantly increased sensitivity compared to directly using Pearson's correlation as an FC prior (Fig. 2(c)). We speculate that this increase arises from more false correlations and indirect effects being eliminated compared to true correlations on average. Retaining only fiber count with functional support also significantly enhanced sensitivity, which is likely due to spurious tracts being removed. However, when we zeroed out $\hat{\boldsymbol{\Lambda}}_{i j}$ that has no anatomical support, detection sensitivity actually reduced. This suggests that the sparse inverse covariance estimates have false correlations and indirect effects reasonably controlled, thus nulling $\hat{\boldsymbol{\Lambda}}_{i j}$ with no anatomical support actually removed important connections missed by the AC estimates. Hence, determining the presence of a functional connection completely based on AC estimates could be detrimental. Instead, penalizing FC estimates with no anatomical support [1] or probabilistically modeling the absence of anatomical connections [2] are likely better strategies for FC-AC integration.

\section{Conclusions}

We showed on a cohort of 60 subjects that FC and AC estimates are not very consistent for typical RS-fMRI and dMRI data. Integrating them as priors for task activation detection further demonstrated their inconsistencies. Importantly, we illustrated that isolating fibers with functional support as well as removing Pearson's correlation with no anatomical support enhanced activation detection compared to directly using fiber count and Pearson's correlation as priors. This suggests that the inconsistencies actually make integration of FC and AC estimates all the more valuable for connectivity estimation. However, when $\hat{\Lambda}_{i j}$ with no anatomical support was nulled, activation detection worsened. Thus, FC and AC estimates should be combined with caution. 
Acknowledgements. This work was supported by the Genim ANR-10-BLAN-0128 grant, NSERC, and the Berkeley-INRIA-Stanford grant. The data were acquired within the IMAGEN project. Jean Baptiste Poline was partly funded by the IMAGEN project, which receives funding from the E.U. Community's FP6, LSHM-CT-2007037286. This manuscript reflects only the authors' views and the Community is not liable for any use that may be made of the information contained therein.

\section{References}

1. Ng, B., Varoquaux, G., Poline, J.-B., Thirion, B.: A Novel Sparse Graphical Approach for Multimodal Brain Connectivity Inference. In: Ayache, N., Delingette, H., Golland, P., Mori, K. (eds.) MICCAI 2012, Part I. LNCS, vol. 7510, pp. 707-714. Springer, Heidelberg (2012)

2. Venkataraman, A., Rathi, Y., Kubicki, M., Westin, C.F., Golland, P.: Joint Modeling of Anatomical and Functional Connectivity for Population Studies. IEEE Trans. Med. Imaging 31, 164-182 (2012)

3. Damoiseaux, J.S., Greicius, M.D.: Greater than the Sum of its Parts: A Review of Studies Combining Structural Connectivity and Resting-state Functional Connectivity. Brain Struct. Funct. 213, 525-533 (2009)

4. Honey, C.J., Sporns, O., Cammoun, L., Gigandet, X., Thiran, J.P., Meuli, R., Hagmann, P.: Predicting Human Resting-state Functional Connectivity from Structural Connectivity. Proc. Nat. Acad. Sci. 106, 2035-2040 (2009)

5. Skudlarski, P., Jagannathan, K., Calhoun, V.D., Hampson, M., Skudlarska, B.A., Pearlson, G.: Measuring Brain Connectivity: Diffusion Tensor Imaging Validates Resting State Temporal Correlations. NeuroImage 43, 554-561 (2008)

6. Marrelec, G., Krainik, A., Duffau, H., Pelegrini-Issac, M., Lehericy, S., Doyon, J., Benali, H.: Partial Correlation for Functional Brain Interactivity Investigation in Functional MRI. NeuroImage 32, 228-237 (2006)

7. Jbabdi, S., Johansen-Berg, H.: Tractography: Where Do We Go from Here? Brain Connectivity $1,169-183$ (2011)

8. Hsieh, C.J., Sustik, M.A., Dhillon, I.S., Ravikumar, P.: Sparse Inverse Covariance Matrix Estimation Using Quadratic Approximation. In: Advances in Neural Information Processing Systems, vol. 24, pp. 2330-2338 (2011)

9. Ng, B., Abugharbieh, R., Varoquaux, G., Poline, J.B., Thirion, B.: Connectivity-informed fMRI Activation Detection. In: Fichtinger, G., Martel, A., Peters, T. (eds.) MICCAI 2011, Part II. LNCS, vol. 6892, pp. 285-292. Springer, Heidelberg (2011)

10. Nichols, T., Hayasaka, S.: Controlling the Familywise Error Rate in Functional Neuroimaging: a Comparative Review. Stat. Methods Med. Research 12, 419-446 (2003)

11. Pinel, P., Thirion, B., Meriaux, S., Jober, A., Serres, J., Le Bihan, D., Poline, J.B., Dehaene, S.: Fast Reproducible Identification and Large-scale Databasing of Individual Functional Cognitive Networks. BioMed. Central Neurosci. 8, 91 (2007)

12. Van Essen, D.C., Ugurbil, K.: The Future of the Human Connectome. NeuroImage 62, 1299-1310 (2012)

13. Malcolm, J.G., Shenton, M.E., Rathi, Y.: Filtered Multitensor Tractography. IEEE Trans. Med. Imaging 29, 1664-1675 (2010) 